\title{
Dynamics of a two-state system through a real level crossing
}

\author{
Benedetto D. Militello ${ }^{1}$ and Nikolay V. Vitanov ${ }^{2}$ \\ ${ }^{1}$ Dipartimento di Fisica e Chimica, Università degli Studi di Palermo, Via Archirafi 36, I-90123 Palermo, Italy \\ ${ }^{2}$ Department of Physics, St Kliment Ohridski University of Sofia, 5 James Bourchier Blvd, 1164 Sofia, Bulgaria
}

\begin{abstract}
The dynamics of a two-state system whose energies undergo a real crossing at some instant of time is studied. At this instant, both the coupling and the detuning vanish simultaneously, which leads to an exact degeneracy of the eigenenergies of the system. It is found that the dynamics of the system is primarily determined by the manner in which the degeneracy occurs. This interesting behavior is reminiscent of a symmetry breaking process, since the totally symmetric situation occurring at the crossing is significantly altered by infinitesimal quantities, which remove the degeneracy, with very important dynamical implications from there on. A very simple analytical formula is derived, which is found to describe the population changes very accurately.
\end{abstract}

PACS numbers: $32.80 . \mathrm{Xx}, 32.80 . \mathrm{Qk}, 33.80 . \mathrm{Be}, 42.50 . \mathrm{Dv}$,

\section{INTRODUCTION}

In the realm of adiabatic evolution [1], the dynamics of a two-state system whose energies cross at some point plays an important role. The exactly solvable model of Landau-Zener-Stueckelberg-Majorana (LZSM) 2 [5] is the most famous example of such class of problems. Its widespread applications range from quantum optics and atomic physics to chemistry and biophysics. In fact, this model is of interest in several physical systems [6], such as Josephson junctions [7, 8], cold atoms in optical lattices [9] and spinorial Bose-Einstein condensates [10]. In order to better consider realistic situations, the implications of having a finite duration [11, 12] or a nonlinear crossing [13] in the experiments have also been studied. Generalizations of the original model to the cases wherein more than two states are involved have been considered [14 16], as well as level crossings occurring in systems described by nonlinear equations [17]. The relevance of the LZSM model in statistical physics, especially in connection with phase transitions in the Ising model, has been discussed [18, 19]. Moreover, recently, a geometric Landau-Zener-Stückelberg interferometer has been realized in single trapped ions by Zhang et al [20] on the basis of a proposal by Gasparinetti et al [21], and LandauZener transitions in frozen pairs of Rydberg atoms have been observed by Saquet et al 22].

In the original LZSM model, the bare energy levels of a two-state system cross at some instant of time, but, due to the presence of a (constant) interaction, the degeneracy is removed in the adiabatic eigenenergies. Then the adiabatic following of an instantaneous eigenstate of the Hamiltonian leads to a perfect transition from one bare state to the other. Beyond the perfect adiabaticity, the correction to the dynamics is given by the formula found by Landau, Zener, Stückelberg and Majorana which expresses the transition probability between the eigenstates (non-adiabatic transitions) of the Hamiltonian [2 [5], and which is equal to the no-transition probability between the bare states.

In 1990 Fishman et al have introduced a variant of the original LZSM model where neither the bare energies nor the dressed ones really cross [23], showing that, even in the presence of the same dressed energies, the circumstance that the bare energies do not cross as in the standard model determines significatively different results. One of the most interesting experimental realizations of this modified LZSM model has been provided by Bouwmeester et al 24], who have observed such modified Landau-Zener dynamics (they call the relevant mathematical description the 'hidden-crossing model') in classical optical systems. By the way, it is worth mentioning that with their experiment Bouwmeester et al have demonstrated that the validity of the LZSM model (and related ones) is not limited to the realm of quantum mechanics.

The variant of the LZSM model complementary to the hidden-crossing model corresponds to a physical situation where both bare and dressed energies cross at the same time, which has never been studied. It is then of interest to analyze the adiabatic evolution of a two-state system in the special case where a real degeneracy occurs at the crossing of the bare energies. In other words, we want to study the case where at the same time when the bare energies cross, the interaction, assumed non vanishing in the rest of the process, vanishes too. At this instant the adiabatic (i.e., dressed) energies, just like the diabatic (i.e., bare) energies, become degenerate. In this case the LZSM formula is not applicable, and in order to estimate the transition probability we have to scrutinize the intricate behavior of the eigenenergies and the nonadiabatic coupling in the vicinity of this real crossing.

The paper is organized as follows. In the next section, we introduce the model Hamiltonian we are going to analyze. In section [II we discuss the condition for the validity of the adiabatic approximation, firstly in general and secondly focusing on the instant of time when the level crossing occurs. In section IV we report on an analysis that allows to predict the efficiency of population transfer on the basis of the peculiarities of the Hamiltonian. Finally, in section $\mathrm{V}$ we summarize the results and provide some conclusive remarks. 


\section{THE MODEL}

Consider a two-state system whose dynamics is governed by a Hamiltonian having the following form $(\hbar=$ $1)$ :

$$
H=\frac{\Delta}{2} \sigma_{z}+\frac{\Omega}{2} \sigma_{x}=\frac{1}{2}\left(\begin{array}{cc}
-\Delta & \Omega \\
\Omega & \Delta
\end{array}\right)
$$

This Hamiltonian has eigenvalues

$$
E_{ \pm} \equiv \omega_{ \pm}= \pm \frac{\Omega}{2} \sqrt{1+\alpha^{2}}
$$

and eigenstates

$$
\begin{aligned}
& \left|\psi_{+}\right\rangle=\cos \theta|\downarrow\rangle+\sin \theta|\uparrow\rangle, \\
& \left|\psi_{-}\right\rangle=\sin \theta|\downarrow\rangle-\cos \theta|\uparrow\rangle,
\end{aligned}
$$

where

$$
\begin{aligned}
\tan \theta & =\alpha+\sqrt{1+\alpha^{2}}, \\
\alpha & =\frac{\Delta}{\Omega} .
\end{aligned}
$$

In the LZSM model, the interaction is constant $(\dot{\Omega}=0)$ and the bare energies change linearly $(\Delta=\kappa t)$. If the change is very slow ( $\kappa$ small enough) then the evolution is adiabatic. Moreover, when $t$ varies from $-\infty$ to $+\infty$, the dressed states change from one bare state to the other; for example, $\left|\psi_{+}(-\infty)\right\rangle=|\downarrow\rangle$ becomes $\left.\left|\psi_{+}(\infty)\right\rangle=|\uparrow\rangle\right)$. Therefore, if the system is prepared in one of the bare states, and the system follows the corresponding dressed state, then in the end of the process each bare state is perfectly mapped to the other bare state: population inversion has occurred. Perfect adiabatic evolution does not exist (except for constant Hamiltonian or for $\Delta \equiv 0$ ), and the LZSM formula gives the deviation from perfect population inversion.

In the following, we will instead study the adiabatic evolution when $\Omega$ and $\Delta$ are such that $\Delta \rightarrow 0$ and $\Omega \rightarrow 0$, when $t \rightarrow 0$, so that a real level crossing occurs at the time instant $t=0$. Of course, there are several ways the two quantities can approach zero, and depending on this, the evolution of the system can be very different.

In order to maintain the possibility of having a complete population transfer, we will always assume that $\alpha$ varies from $-\infty$ (i.e., a huge negative value) to $+\infty$ (a huge positive value) or vice versa. Indeed, this will make each of the dressed states change from being exactly (i.e., essentially) equal to one bare state to being exactly (i.e., essentially) equal to the other bare state along the experiment.

\section{ADIABATIC EVOLUTION}

After introducing the unitary operator describing the passage to the basis of the instantaneous eigenstates of the Hamiltonian,

$$
\begin{aligned}
T_{\mathrm{A}} & =\left|\psi_{+}(-\infty)\right\rangle\left\langle\psi_{+}(t)|+| \psi_{-}(-\infty)\right\rangle\left\langle\psi_{-}(t)\right| \\
& =|\downarrow\rangle\left\langle\psi_{+}(t)|+| \uparrow\right\rangle\left\langle\psi_{-}(t)\right|,
\end{aligned}
$$

we easily find that in this basis the evolution is generated by the following operator:

$$
-\mathrm{i} T_{\mathrm{A}} \dot{T}_{\mathrm{A}}^{\dagger}+T_{\mathrm{A}} H T_{\mathrm{A}}^{\dagger}=\left(\begin{array}{cc}
\omega_{+} & \mathrm{i} \dot{\theta} \\
-\mathrm{i} \dot{\theta} & \omega_{-}
\end{array}\right) .
$$

It is worth noting that for $\dot{\theta}=0$ one would seemingly have perfect adiabatic evolution (in fact, each of the two instantaneous eigenstates would just acquire a phase, without undergoing any transitions). But, if $\dot{\theta}=0$ then there is no change of the eigenstates of the Hamiltonian, and then there is no real adiabatic evolution. In fact, the adiabatic following of the eigenstates of the Hamiltonian governing the system is intrinsically the result of some approximations and obtaining an exact adiabatic following of the eigenstates of the system Hamiltonian is a chimera. There is, seemingly, a violation to such an assertion given by the shortcuts to adiabaticity [25 27]. In fact, such techniques allow to realize the exact adiabatic following of the eigenstates of a given Hamiltonian. But the crucial point of such shortcuts is that the exact following is obtained through an additional interaction, so that, in the end, the adiabatic evolution does not coincide with the adiabatic following of the eigenstates of the system Hamiltonian. In other words, in order to realize the adiabatic following of the eigenstates of a given Hamiltonian $H(t)$ it is necessary to let the system evolve under the action of $H(t)+H_{s c}(t)$, where $H_{s c}(t)$ is a suitable additional term that allows to realize the shortcut and which does not commute with $H(t)$. In this way, there is no following of the eigenstates of the total Hamiltonian of the system $H(t)+H_{s c}(t)$.

\section{A. General condition for adiabaticity}

In order to have an adiabatic evolution the terms responsible for transitions between the eigenstates of the Hamiltonian must be negligible, which in our case means: $\eta \equiv|\dot{\theta}| /\left|\omega_{+}-\omega_{-}\right| \ll 1$. Now, since

$$
\dot{\theta}=\frac{\alpha+\sqrt{1+\alpha^{2}}}{1+\left(\alpha+\sqrt{1+\alpha^{2}}\right)^{2}} \frac{\dot{\alpha}}{\sqrt{1+\alpha^{2}}}
$$

one finds:

$$
\begin{aligned}
\eta & =|\dot{\theta}| /\left|\omega_{+}-\omega_{-}\right| \\
& =\left|\frac{\alpha+\sqrt{1+\alpha^{2}}}{1+\left(\alpha+\sqrt{1+\alpha^{2}}\right)^{2}}\right| \times\left|\frac{\dot{\alpha}}{\Omega\left(1+\alpha^{2}\right)}\right|,
\end{aligned}
$$

which can also be cast in the following form (consider that $\alpha+\sqrt{1+\alpha^{2}}=1 /\left(-\alpha+\sqrt{1+\alpha^{2}}\right)$ and then $\left.2 \sqrt{1+\alpha^{2}}=\tan \theta+1 / \tan \theta\right)$ :

$$
\eta=|\sin (2 \theta)|^{3} \times|\dot{\alpha} / \Omega| .
$$


Putting $\eta$ in this form, it is clear that a sufficient condition to guarantee an adiabatic evolution is $|\dot{\alpha} / \Omega| \ll 1$. Nevertheless, in some cases, a finite or even a divergent value of $|\dot{\alpha} / \Omega|$ can be compensated by the smallness of $\sin (2 \theta)$.

Let us come back to Eq. (8) and consider what the condition for adiabaticity becomes in the cases $|\alpha| \ll 1$, $\alpha \sim 1$ and $|\alpha| \gg 1$. First of all, consider that both for $|\alpha| \ll 1$ and $\alpha \sim 1$ one can say that $\eta \sim|\dot{\alpha} / \Omega|=\mid(\dot{\Omega} \Delta-$ $\Omega \dot{\Delta}) / \Omega^{3} \mid$, while for $|\alpha| \gg 1$ one has $\eta \sim\left|\dot{\alpha} /\left(\alpha^{3} \Omega\right)\right|=$ $\left|(\dot{\Omega} \Delta-\Omega \dot{\Delta}) / \Delta^{3}\right|$. Therefore, the conditions to have an adiabatic evolution become:

$$
\begin{aligned}
& \left\{\begin{array}{l}
|\alpha| \ll 1 \\
|\alpha| \sim 1 \Rightarrow\left|(\dot{\Omega} \Delta-\Omega \dot{\Delta}) / \Omega^{3}\right| \ll 1,
\end{array}\right. \\
& |\alpha| \gg 1 \Rightarrow\left|(\dot{\Omega} \Delta-\Omega \dot{\Delta}) / \Delta^{3}\right| \ll 1 .
\end{aligned}
$$

Note that when $|\alpha| \gg 1$, for $\alpha>0$ one has $\theta \rightarrow \pi / 2$, while for $\alpha<0$ it turns out $\theta \rightarrow 0$, and then $\sin (2 \theta) \rightarrow 0$ in both cases. This is just the case mentioned above where smallness of $\sin (2 \theta)$ can compensate higher values of $|\dot{\alpha} / \Omega|$, and this is the reason why the condition for having an adiabatic evolution assumes a different form.

\section{B. Adiabaticity at the level crossing}

It is of interest to give some general indication of the validity of the adiabatic approximation close to the level crossing. In order to develop such an analysis, let us consider the behavior of $\Delta(t)$ and $\Omega(t)$ close to the level crossing occurring at $t=0$, assuming that the leading terms in their Taylor expansions versus $t$ at the crossing $(t=0)$ are

$$
\begin{aligned}
& \Delta \simeq A t^{a}, \\
& \Omega \simeq B t^{b},
\end{aligned}
$$

with $a, b>0$. Then, in the case $\alpha \ll 1(a>b)$, one has:

$$
\eta \sim\left|\frac{A(b-a)}{B^{2}}\right| \times|t|^{a-2 b-1},
$$

and to guarantee $\eta \ll 1$ one needs $a>2 b+1$.

In the case $\alpha \gg 1(b>a)$ it turns out,

$$
\eta \sim\left|\frac{A(b-a)}{B^{2}}\right| \times|t|^{b-2 a-1} .
$$

and to guarantee $\eta \ll 1$ one needs $a<(b-1) / 2$.

Therefore, each of the following inequalities guarantee adiabaticity in the very neighborhood of $t=0$ :

$$
\begin{aligned}
& a>2 b+1, \\
& b>2 a+1 .
\end{aligned}
$$

The last case to consider is given by the situation $|\alpha| \sim$ 1 , which deserves a specific analysis since it requires the analysis of further terms in the Taylor expansion of both $\Delta(t)$ and $\Omega(t)$.

\section{NONADIABATIC TRANSITIONS}

In this section we give some general dynamical properties that allow to forecast the efficiency of the population transfer on the basis of an analysis of $\Delta(t)$ and $\Omega(t)$ at the level crossing.

\section{A. Transitions at the level crossing}

Let us first of all assume that the evolution of the system is essentially adiabatic everywhere, with the only possible exception of the very neighborhood of $t=0$. The total evolution operator (that is, the evolution operator in the Schrödinger picture) can be written as:

$$
\begin{aligned}
U(+\infty,-\infty) & =U\left(+\infty, 0^{+}\right) U\left(0^{+}, 0^{-}\right) U\left(0^{-},-\infty\right) \\
& \approx U_{\mathrm{A}}\left(+\infty, 0^{+}\right) U\left(0^{+}, 0^{-}\right) U_{\mathrm{A}}\left(0^{-},-\infty\right)
\end{aligned}
$$

where we have introduced the adiabatic evolution operator:

$$
U_{\mathrm{A}}\left(t_{2}, t_{1}\right)=\sum_{k= \pm} e^{-\mathrm{i} \varphi_{k}\left(t_{2}, t_{1}\right)}\left|\psi_{k}\left(t_{2}\right)\right\rangle\left\langle\psi_{k}\left(t_{1}\right)\right|,
$$

with

$$
\varphi_{k}\left(t_{2}, t_{1}\right)=\int_{t_{1}}^{t_{2}}\left(\omega_{k}(s)-\mathrm{i}\left\langle\psi_{k}(s) \mid \dot{\psi}_{k}(s)\right\rangle\right) \mathrm{d} s .
$$

Now, since around $t=0$ the Hamiltonian $H$ does not exhibit singularities (far from this, it is infinitesimal), one has $U\left(0^{+}, 0^{-}\right) \approx \mathbf{I}$, and then:

$$
U(+\infty,-\infty) \approx U_{\mathrm{A}}\left(+\infty, 0^{+}\right) U_{\mathrm{A}}\left(0^{-},-\infty\right)
$$

from which:

$$
U(+\infty,-\infty)=\sum_{k= \pm} \sum_{l= \pm} T_{k l}\left|\psi_{k}(+\infty)\right\rangle\left\langle\psi_{l}(-\infty)\right|
$$

where

$$
\begin{aligned}
& T_{k l}=\mathrm{e}^{-\mathrm{i}\left(\varphi_{k}^{-}+\varphi_{l}^{+}\right)}\left\langle\psi_{k}\left(0^{+}\right) \mid \psi_{l}\left(0^{-}\right)\right\rangle, \\
& \varphi_{k}^{-}=\varphi_{k}\left(0^{-},-\infty\right), \\
& \varphi_{l}^{+}=\varphi_{k}\left(+\infty, 0^{+}\right) .
\end{aligned}
$$

Now, consider what follows:

$$
\begin{aligned}
& \left\langle\psi_{ \pm}(s) \mid \dot{\psi}_{ \pm}(s)\right\rangle=0, \\
& \left\langle\psi_{ \pm}(s) \mid \dot{\psi}_{\mp}(s)\right\rangle= \pm \dot{\theta}
\end{aligned}
$$

and, more important,

$$
\begin{aligned}
\left\langle\psi_{+}\left(0^{+}\right) \mid \psi_{+}\left(0^{-}\right)\right\rangle & =\cos \Theta, \\
\left\langle\psi_{-}\left(0^{+}\right) \mid \psi_{-}\left(0^{-}\right)\right\rangle & =\cos \Theta, \\
\left\langle\psi_{+}\left(0^{+}\right) \mid \psi_{-}\left(0^{-}\right)\right\rangle & =-\sin \Theta, \\
\left\langle\psi_{-}\left(0^{+}\right) \mid \psi_{+}\left(0^{-}\right)\right\rangle & =\sin \Theta,
\end{aligned}
$$


with

$$
\Theta=\theta\left(0^{+}\right)-\theta\left(0^{-}\right) .
$$

From Eqs. (21) we can derive the probabilities of the nonadiabatic transitions. The failure of adiabatic passage in the overall process can be quantified through the parameter $\Theta$ :

$$
\begin{aligned}
& \left|\left\langle\psi_{ \pm}(+\infty)|U(+\infty,-\infty)| \psi_{ \pm}(-\infty)\right\rangle\right|^{2}=\cos ^{2} \Theta, \\
& \left|\left\langle\psi_{ \pm}(+\infty)|U(+\infty,-\infty)| \psi_{\mp}(-\infty)\right\rangle\right|^{2}=\sin ^{2} \Theta .
\end{aligned}
$$

The first of such two equations, Eq. 23a , says that each of the two eigenstates of $H,\left|\psi_{+}(t)\right\rangle$ or $\left|\psi_{-}(t)\right\rangle$, follows the adiabatic change of the Hamiltonian with probability $\cos ^{2} \Theta$. On the other hand, Eq. (23b) says that each of such two states undergoes a transition to the other state with probability $\sin ^{2} \Theta$.

The nonadiabatic transitions are determined by the abrupt change of the eigenstates of the Hamiltonian at $t=0$. It is interesting to point out that, from the dynamical point of view, nothing really happens around $t=0$, because the Hamiltonian is essentially zero in that time interval. Nevertheless, what happens from there on is mainly determined by the possible abrupt change of the Hamiltonian eigenstates occurring at $t=0$.

The case $\Theta=0$ can be obtained when $\alpha \rightarrow 0$, which implies $\theta\left(0^{+}\right)=\theta\left(0^{-}\right)=\pi / 4$. On the contrary, the case $\Theta=\pi / 2(-\pi / 2)$ can be obtained when $\alpha \rightarrow-\infty(+\infty)$ on the left of $t=0$ and $\alpha \rightarrow+\infty(-\infty)$ on the right. Therefore, we can say that when $\Delta$ is infinitesimal of higher order than $\Omega$ there is no abrupt change of the eigenstates and then there is a total transfer of population. On the other hand, when $\Omega$ is infinitesimal of higher order than $\Delta$ we expect an abrupt change of the eigenstates and then a very low population transfer efficiency. When $\Delta$ and $\Omega$ are infinitesimals of the same order, a specific analysis of the behavior of $\theta$ is necessary.

All these results have been derived under the assumption that the adiabatic approximation is valid everywhere far from $t=0$, and that a possible critical behavior can occur only when the levels cross $(t=0)$.

In the standard treatment of Landau-ZenerStueckelberg-Majorana processes, $\Omega$ is assumed to be constant and $\Delta$ is considered as a linear function of time. In our examples, both for the sake of simplicity and to make it easier to compare our situations with the standard one, we have always assumed $\Delta=\kappa t$, with $\kappa$ being a suitable parameter.

In Fig. 1 we show the population transfer efficiency (i.e., the final population of the initially unpopulated state) when the $\Omega$-pulse is essentially constant except for the neighborhood of the level crossing: $\Omega=\Omega_{0}(1-$ $\exp (-|t / \sigma|))$. The parameter $\sigma$ measures the size of the region where $\Omega$ mainly varies. In Fig. 2 we consider a case where the shape of $\Omega$ is far from being constant: $\Omega=\lambda \Omega_{0} \sin (\pi t / T)$, where $T$ represents the duration of the whole process, while $\lambda$ determines the maximum values of the pulse. In both Figs. 1 and 2 a very good

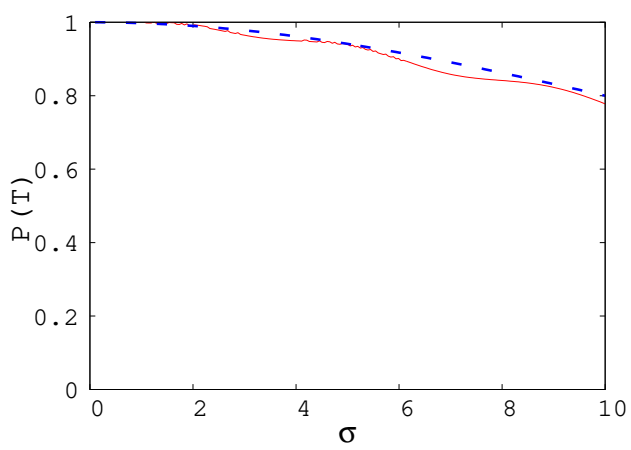

(a)

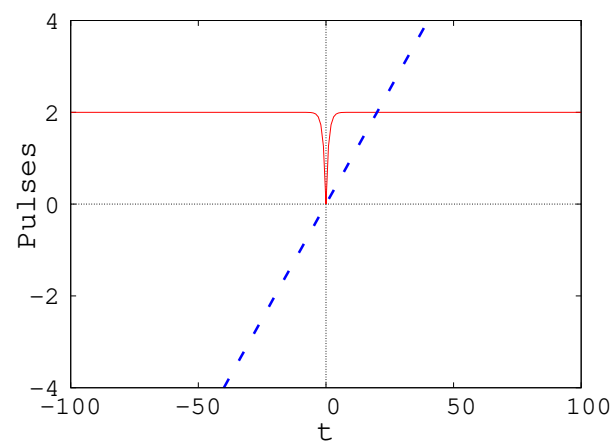

(b)

FIG. 1: (Color online) Frame (a) shows the asymptotic populations of the bare state $|\uparrow\rangle$ (red solid line) and the theoretical prediction of the efficiency (blue dashed line), $\cos ^{2} \Theta$, vs the parameter $\sigma$ (in units of $\left.2 / \Omega_{0}\right)$. Here $\Omega=\Omega_{0}(1-\exp (-|t / \sigma|)$ ), $\Delta=\kappa t$, with $k / \Omega_{0}^{2}=0.025$ and $\kappa T / \Omega_{0}=50$, where $2 T$ is the duration of the experiment. Frame (b) shows the coupling $\Omega$ (red solid line) and the detuning $\Delta$ (blue dashed line) for $\sigma=2 / \Omega_{0}$. The amplitudes of the pulses are in units of $\Omega_{0} / 2$ and time is in units of $2 / \Omega_{0}$.

agreement between the results from the numerical analysis and the theoretical predictions $\left(\cos ^{2} \Theta\right)$ is evident.

Figure 3 compares numerical and analytical results for $\Omega=\lambda \beta t \sin (\pi t / T)$. We observe nearly zero population transfer efficiency, which is due to the fact that $\alpha=\Delta / \Omega$ diverges at $t=0$, assuming negative values for $t<0$ and positive values on the other side, which makes $\theta\left(0^{-}\right)=0$ and $\theta\left(0^{+}\right)=\pi / 2$. The numerical results and theoretical predictions have a very good agreement also this time. Of course, in order to support the applicability of our theoretical analysis, we have always carefully checked (through numerical evaluation of $\eta$ ) that the adiabatic approximation is valid essentially everywhere, with the only possible exception of $t=0$ and its vicinity.

\section{B. Transitions around the level crossing}

It can happen that the validity of the adiabatic approximation is broken before reaching the level crossing and for a while after it has occurred. In such a case, assuming that it is valid out of the (small) time interval 


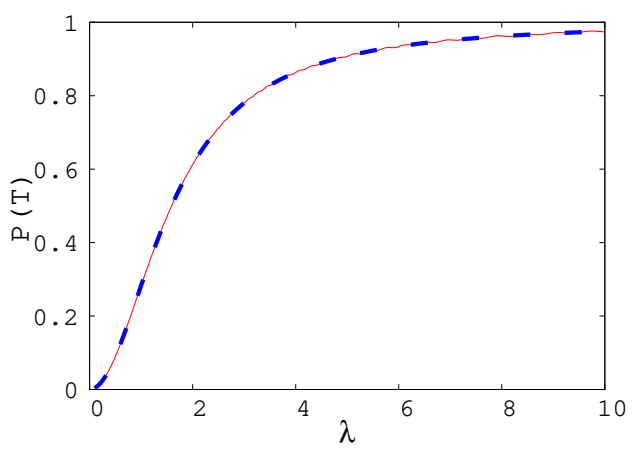

(a)

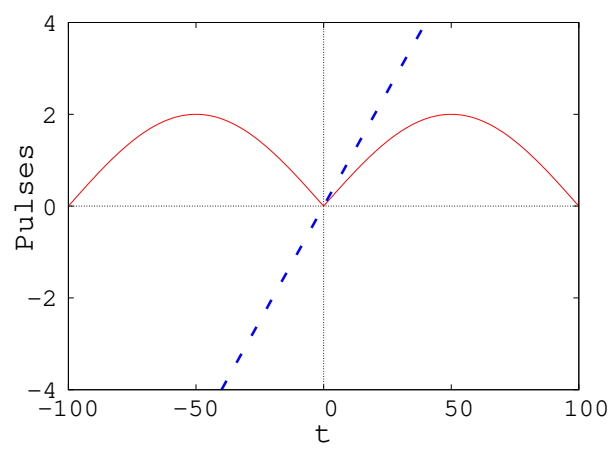

(b)

FIG. 2: (Color online) Frame (a) shows the asymptotic populations of the bare state $|\uparrow\rangle$ (red solid line) and the theoretical prediction of the efficiency (blue dashed line), $\cos ^{2} \Theta$, vs the parameter $\lambda$ (dimensionless). Here $\Omega=\lambda \Omega_{0} \sin (\pi t / T)$, where $2 T$ is the duration of the experiment, and $\Delta=\kappa t$, with $k / \Omega_{0}^{2}=0.025$ and $\kappa T / \Omega_{0}=5$. Frame (b) shows the coupling $\Omega$ (red solid line) and the detuning $\Delta$ (blue dashed line) for $\lambda=1$. The amplitudes of the pulses are in units of $\Omega_{0} / 2$ and time is in units of $2 / \Omega_{0}$.

$[-\tau, \tau]$, one can write:

$$
\begin{aligned}
U(+\infty,-\infty) & =U(+\infty, \tau) U(\tau,-\tau) U(-\tau,-\infty) \\
& \approx U_{\mathrm{A}}(+\infty, \tau) U(\tau,-\tau) U_{\mathrm{A}}(-\tau,-\infty)
\end{aligned}
$$

Since $\tau$ is very small and assuming that $H$ does not exhibit singularities at any time, we can make the following (rough) approximation:

$$
\begin{aligned}
U(\tau,-\tau) & \approx \mathbf{I}-\mathrm{i} \int_{-\tau}^{\tau} H(s) \mathrm{d} s \\
& \approx \exp \left(-\mathrm{i} \int_{-\tau}^{\tau} H(s) \mathrm{d} s\right) .
\end{aligned}
$$

If $\Delta$ is an odd function, then one easily obtains:

$$
U(\tau,-\tau) \approx \cos \xi \mathbf{I}-\mathrm{i} \sin \xi \sigma_{x}, \quad \xi=\int_{-\tau}^{\tau} \Omega(s) \mathrm{d} s
$$

Therefore, assuming adiabaticity everywhere out of the interval $[-\tau, \tau]$, the efficiency of the population transfer

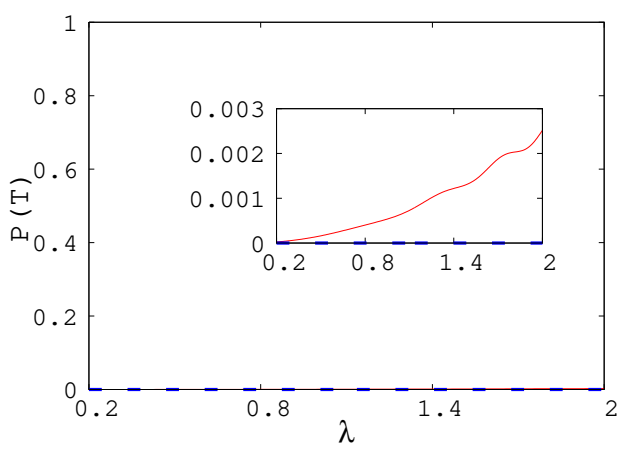

(a)

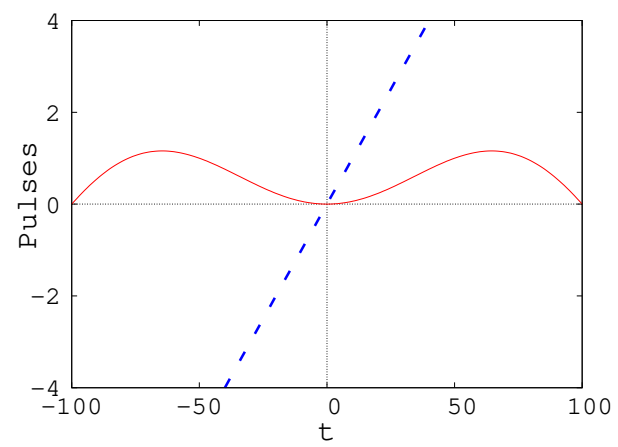

(b)

FIG. 3: (Color online) Frame (a) shows the asymptotic populations of the bare state $|\uparrow\rangle$ (red solid line) and the theoretical prediction of the efficiency (blue dashed line), $\cos ^{2} \Theta$, vs the parameter $\lambda$ (dimensionless). The inset is a 'zoom' that shows the same quantities in a different $y$-range. Here $\Omega=\lambda \beta t \sin (\pi t / T)$, where $2 T$ is the duration of the experiment, and $\Delta=\kappa t$, with $\kappa^{2} / \beta=5$ and $\beta T^{2}=200$. Frame (b) shows the coupling $\Omega$ (red solid line) and the detuning $\Delta$ (blue dashed line) for $\lambda=1$. The amplitudes of the pulses are in units of $\sqrt{\beta / 2}$ and time is in units of $\sqrt{2 / \beta}$.

could be roughly given by:

$$
\begin{aligned}
& \left|\left\langle\psi_{ \pm}(\tau)|U(\tau,-\tau)| \psi_{ \pm}(-\tau)\right\rangle\right|^{2} \\
& \quad \approx \cos ^{2} \xi \cos ^{2}[\theta(\tau)-\theta(-\tau)] \\
& \quad+\sin ^{2} \xi \sin ^{2}[\theta(\tau)+\theta(-\tau)]
\end{aligned}
$$

If the adiabaticity is broken far from the level crossing then all this treatment is not able to describe the behavior of the system. On the other hand, even under the proper hypotheses about adiabaticity, Eq. (27) may give a wrong prediction because the first order approximation to evaluate $U(-\tau, \tau)$ can be insufficient. In this case, further terms of the relevant Dyson expansion have to be used.

\section{DISCUSSION}

In this paper we have analyzed the dynamics of a twostate system whose energies undergo a level crossing. This is a kind of situation that cannot be treated by the 
LZSM model, because the interaction between the two states approaches zero when the bare energies cross.

The theoretical analysis makes it possible to predict the transition probability from one bare state to the other on the basis of the way the bare energies and the intensity of the interaction between them approach zero at the same time. Indeed, under the assumption that the adiabatic approximation is valid essentially everywhere except, possibly, around the crossing, the efficiency is mainly affected by the possible abrupt changes of the eigenstates of the Hamiltonian. In fact, if the bare energies cross faster than the interaction there is no abrupt change of the eigenstates and the population transfer efficiency tends to unity, while in the opposite case there is the most dramatic abrupt change of the eigenstates (each eigenstate immediately after the crossing is orthogonal to the corresponding eigenstate just before the crossing) and the efficiency tends to zero. If $\Delta$ and $\Omega$ approach zero as infinitesimal of the same order, then a specific analysis is required and the exact value of their ratio determines the efficiency of the population transfer.

Our theoretical analysis is supported by numerical calculations. We have always found a good agreement between the theoretically predicted efficiency and the corre- sponding numerical result, and in some cases this agreement is perfect.

It is worth noting that in the very neighborhood of the level crossing nothing really happens, from the dynamical point of view, in the sense that the state of the quantum system essentially does not evolve (being $H \approx 0$ ), but what happens at the level crossing significantly affects the dynamics from there on. This circumstance is typical of any sudden change of the Hamiltonian, but in our case the peculiarity is that such a change is determined by infinitesimal quantities and the way they compare each other. Somehow, it resembles a symmetry breaking, since the perfectly symmetric situation at the crossing $(H=$ 0 ) is significantly altered, with important consequences on the system, by very small perturbations such as the pulses around $t=0$.

\section{Acknowledgements}

This work has been financially supported by the Co.R.I. program of the University of Palermo.
[1] A. Messiah, Quantum Mechanics (Dover)

[2] L. D. Landau, Phys. Z. Sowjetunion 2, 46 (1932).

[3] C. Zener, Proc. R. Soc. Lond. Ser. A 137, 696 (1932).

[4] E. C. G. Stueckelberg, Helv. Phys. Acta 5, 369 (1932).

[5] E. Majorana, Nuovo Cimento 9, 43 (1932).

[6] S.-N. Shevchenko, S. Ashahab, and F. Nori, Phys. Rep. 492, 1 (2010).

[7] D.M. Berns, et al., Nature (London) 455, 51 (2008).

[8] G. Sun, et al., Nature Commun. 1, 51 (2010).

[9] A. Zenesini, D. Ciampini, O. Morsch, and E. Arimondo, Phys. Rev. A 82, 065601 (2010).

[10] J.-N. Zhang, C.-P. Sun, S. Yi, and F. Nori, Phys. Rev. A 83, 033614 (2011).

[11] N. V. Vitanov and B. M. Garraway, Phys. Rev. A 53, 4288 (1996); ibid. 54, 5458(E) (1996).

[12] N. V. Vitanov, Phys. Rev. A 59, 988 (1999).

[13] N. V. Vitanov and K.-A. Suominen, Phys. Rev. A 59, 4580 (1999).

[14] A. V. Shytov, Phys. Rev. A 70, 052708 (2004).

[15] G. S. Vasilev, S. S. Ivanov and N. V. Vitanov, Phys. Rev. A 75, 013417 (2007).

[16] S. S. Ivanov and N. V. Vitanov, Phys. Rev. A 77, 023406 (2008).

[17] A. Ishkhanyan, M. Mackie, A. Carmichael, P. L. Gould, and J. Javanainen, Phys. Rev. A 69, 043612 (2004).

[18] A. del Campo, M. M. Rams and W. H. Zurek, Phys. Rev. Lett. 109, 115703 (2012).

[19] N. A. Sinitsyn, Phys. Rev. A 87, 032701 (2013).

[20] J. Zhang, J. Zhang, X. Zhang and K. Kim, Phys. Rev. A 89, 013608 (2014).

[21] S. Gasparinetti, P. Solinas and J. P. Pekola, Phys. Rev. Lett. 107, 207002 (2011).

[22] N. Saquet, A. Cournol, J. Beugnon, J. Robert, P. Pillet and N. Vanhaecke, Phys. Rev. Lett. 104, 133003 (2010).

[23] S. Fishman, K. Mullen and E. Ben-Jacob, Phys. Rev. A 42, 5181 (1990).

[24] D. Bouwmeester, N. H. Dekker, F. E. v. Dorsselear, C. A. Schrama, P. M. Visser and J. P. Woerdman, Phys. Rev. A 51, 646 (1995).

[25] M. V. Berry, J. Phys. A 42365303 (2009).

[26] E. Torrontegui, S.Ibáñez, S. Martínez-Garaot, M. Modugno, A. del Campo, D. Guéry-Odelin, A. Ruschhaupt, Xi Chen, J. Gonzalo Muga, Adv. At. Mol. Opt. Phys. 62, 117 (2013).

[27] A. del Campo and M. G. Boshier, Scientific Reports 2, 648 (2012). 